\title{
Optical Instruments for Local Divertor Observation at Wendelstein 7-X
}

\author{
Olaf Neubauer ${ }^{1}$ \\ E-mail: o.neubauer@fz-juelich.de

\section{Rahim Allahyari, Sebastijan Brezinsek, Guntram Czymek, Peter Denner, Fritz Harberts, Martin Knaup}

Forschungszentrum Jülich GmbH, Institut für Energie- und Klimaforschung - Plasmaphysik, 52425 Jülich, Germany

\section{Ralf König, Maciej Krychowiak, The W7-X Team}

Max Planck Institute for Plasma Physics,

17491 Greifswald, Germany

\section{Bernd Schweer}

Laboratoire de Physique des Plasmas - Laboratorium voor Plasmafysica, ERM/KMS, 1000 Brussels, Belgium

\begin{abstract}
The most advanced stellarator at present - Wendelstein 7-X - is currently commissioned. Forschungszentrum Jülich is contributing a number of diagnostic systems in order to participate in the research field of plasma-wall interactions (PWI). The specific interest at Wendelstein 7-X is to study PWI processes in an island divertor with high density and low temperature at steady state. For local characterization of the divertor plasma, a versatile optical observation system is currently developed. The observation of two opposite divertor locations linked by magnetic field lines is aiming at investigating temperature and density profiles, impurities, hydrogen recycling, and transient heat load deposition. The optical systems consist of two mirror based endoscopes each with overlapping fields of view. The light (wavelength range of $350 \mathrm{~nm}-7000 \mathrm{~nm}$ ) is divided into ultraviolet, visible and infrared components. Both, cameras equipped with narrow band interference filters as well as spectrometers linked via fibre optics are foreseen. The observation includes photon emission induced by interaction of injected gas (He, N, Ne) with plasma, radiation produced by PWI processes of fuel $(\mathrm{H})$ as well as tomographic reconstruction of the impurity $(\mathrm{C}, \mathrm{O}, \mathrm{N}, \mathrm{Ne})$ radiation in the poloidal sections. Finally, the infrared emission from divertor targets will be recorded at temporal resolution of a millisecond.
\end{abstract}

First EPS Conference on Plasma Diagnostics - $1^{\text {st }}$ ECPD

14-17 April 2015,

Villa Mondragone , Frascati (Rome) Italy

\footnotetext{
${ }^{1}$ Speaker 


\section{Introduction}

The most advanced stellarator at present - Wendelstein 7-X [1, 2] - is currently commissioned at Greifswald, Germany, by Max-Planck-Institute for Plasma Physics. The mission of this helical advanced stellarator (HELIAS) type device is to show good energy and particle confinement with highly reduced net-currents in the plasma equilibrium obtained. This route promises an intrinsic stationary confinement of fusion relevant high temperature plasma. A key ingredient for successful operation of Wendelstein 7-X is reliable and stable exhaust of energy and particles to maintain first wall and divertor integrity at low impurity content of the main plasma.

The divertor concept used is the island divertor. It employs magnetic islands in the plasma edge, which represent the interface between core plasma and plasma facing components made of graphite. The qualification of this island divertor concept - later with metallic plasma facing components - is mandatory to explore the capability of a HELIAS type stellarator as a candidate for a future fusion power plant. To tackle this goal, Forschungszentrum Jülich is contributing a number of diagnostic systems [3]. The specific interest at Wendelstein 7-X is to understand plasma-wall interactions (PWI) processes in presence of an island divertor at high density and low temperature $[4,5]$ at steady state operation.

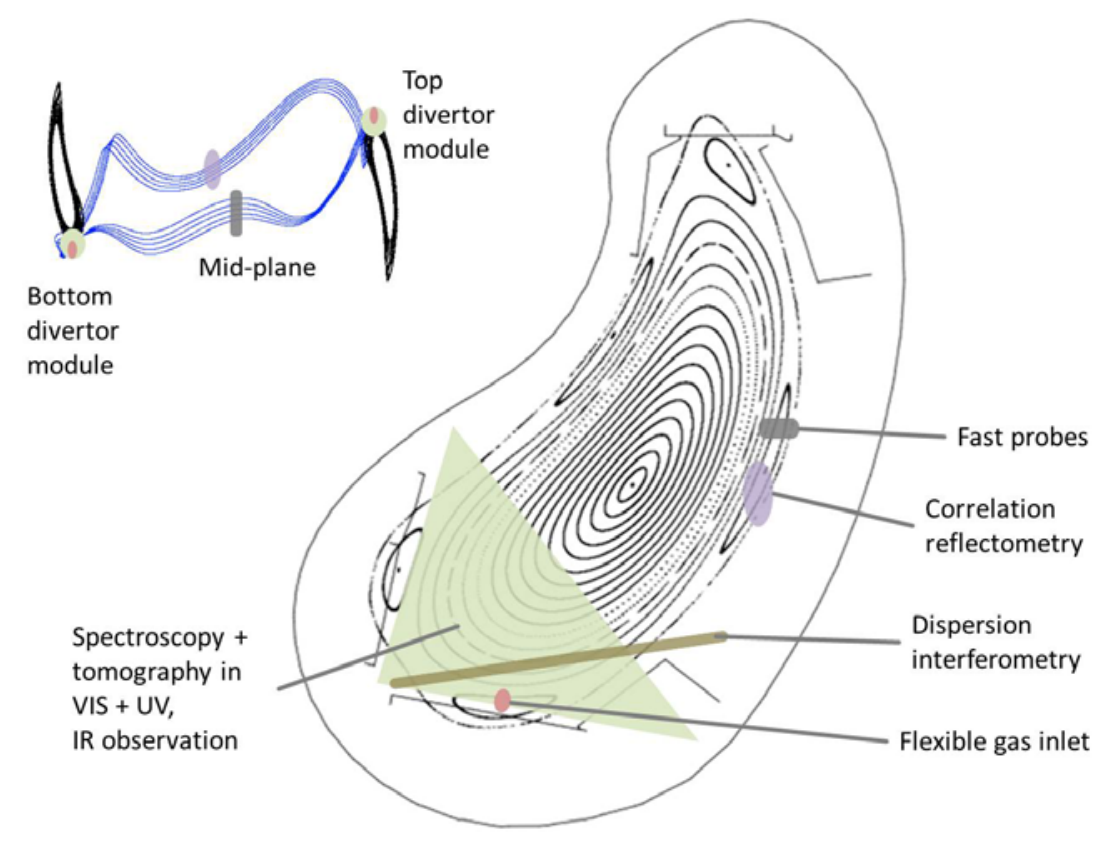

Fig. 1 Configuration of diagnostic systems foreseen for observation of divertor and edge plasma is projected into a single poloidal section (right). Divertor observation will be installed in two plasma sections linked mutually (left). Main chamber interaction systems are linked via magnetic mapping to the same toroidal location (left).

For local characterization of the divertor plasma, a versatile optical observation system is currently developed. Figure 1 gives an overview on the setup foreseen. The observation of two opposite divertor locations linked by magnetic field lines will be assisted by a gas feed system for atomic beam spectroscopy and impurity inlet. Additionally a multipurpose fast probe manipulator for measurement of local profiles, flows and magnetic structures and a poloidal correlation reflectometer for measurement of the local topology will be installed along the same magnetic structure, albeit the latter one between a different divertor pair. 


\section{Measurement requirements}

The particular interest in divertor plasma characterization is to measure temperature and density profiles, impurities, hydrogen recycling, and transient heat load deposition. Profiles of plasma temperature and density in the vicinity of the divertor targets are derived from the characteristic line emission produced by interaction of a dedicated atomic helium beam from the gas feed system with the plasma. For this purpose a spatial resolution comparable to magnetic structures of better than $1 \mathrm{~mm}$ is required in the interaction zone reaching approximately $70 \mathrm{~mm}$ radially into the plasma at the centre of a divertor target. The observation of hydrogen and impurity recycling requires evaluation of characteristic line emission with a field of view covering both divertor plate surfaces. Over the whole depth of field the spatial resolution has to be better than $2 \mathrm{~mm}$. Volume recombination processes expected at high plasma densities have to be identified in a 2D resolution of a divertor section. Finally, transient heat load deposition has to be derived from infrared light emitted from divertor surfaces with a spatial resolution of about $2 \mathrm{~mm}$ and a temporal resolution of approximately $1 \mathrm{~ms}$. Table 1 summarizes important parameters to be fulfilled by the observation system.

\begin{tabular}{lll} 
Property & Unit & Value \\
\hline Spectral range & $\mathrm{nm}$ & $350-7000$ \\
Transmittance & $\%$ & $>30$ \\
Spatial resolution & $\mathrm{mm}$ & $<2$ \\
Object distance & $\mathrm{m}$ & $0.8-0.7$ \\
Depth of field & $\mathrm{m}$ & 0.6 \\
Poloidal and toroidal fields of view & degrees & 9 \\
Poloidal rotation of first mirror & degrees & 30 \\
Aperture size & $\mathrm{mm} * \mathrm{~mm}$ & $4 * 4$ \\
Neutron fluence & $\mathrm{m}^{-2}$ & $1.5 * 10^{18}$ \\
Plasma radiation & $\mathrm{kW} / \mathrm{m}^{2}$ & $<100$
\end{tabular}

Table 1 Measurement requirements and loads for each of the endoscopes.

\section{Endoscope concept}

Three main optical concepts are commonly used for fusion plasma observation: immersion tube, endoscope based on refractive optics, and endoscope based on reflective optics. Immersion tubes by placing a camera behind a vacuum window close to the plasma provide a technically simple solution with high transmittance. However, their flexibility in using several detectors simultaneously is limited. Furthermore, vacuum windows and cameras are subject to neutron damage, and the window itself suffers from carbon deposition in presence of plasma facing components made of carbon. Endoscope systems by transmitting the light to the outside of a fusion device allow for higher flexibility in terms of detector systems and bring window and cameras in safer regions. However, this is paid by higher complexity and lower transmittance compared to immersion tubes. Among endoscopes, those equipped with refractive optic elements still could deteriorate by window coating. Additionally, chromatic aberration prohibits this concept if a large range of wavelength is to be observed. This could be overcome by reflective optics. 


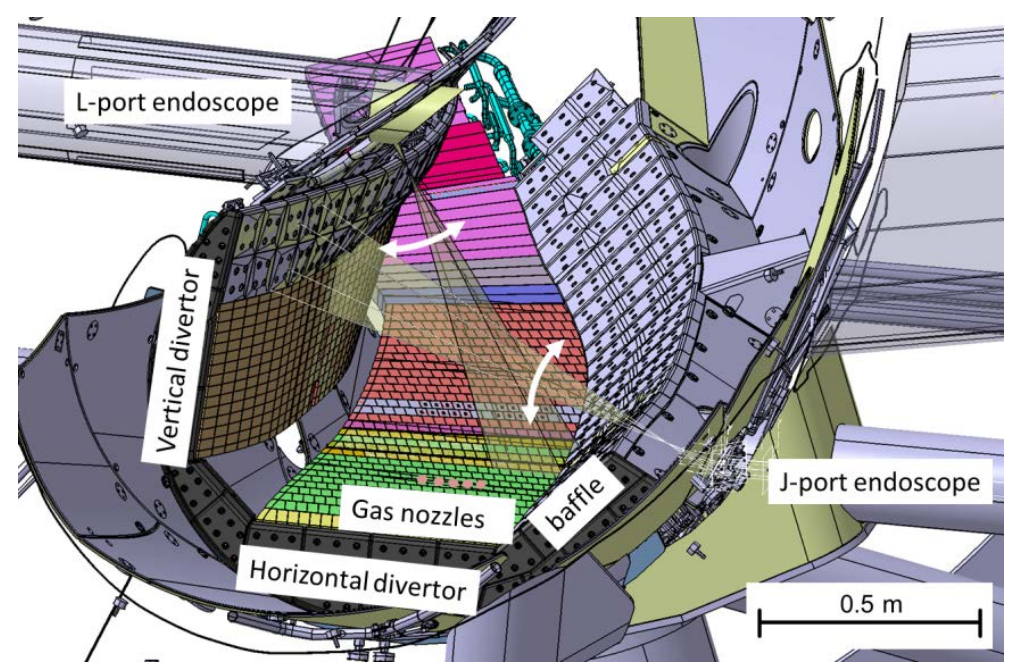

Fig. 2 One out of ten identical divertor modules of Wendelstein 7-X with fields of view of the required local observation system which covers the most loaded section of the divertor.

The optical system chosen for Wendelstein 7-X consists of two endoscopes in a toroidal section with nearly perpendicular fields of view (figure 2) for tomographic reconstruction. Taking into account measurement requirements and environment conditions described above, the concept of mirror based endoscope is the most appropriate if shutters are employed to avoid deposition on the first mirror. With the experience in designing an observation system for the JET divertor [6] this concept (figure 3) was selected.

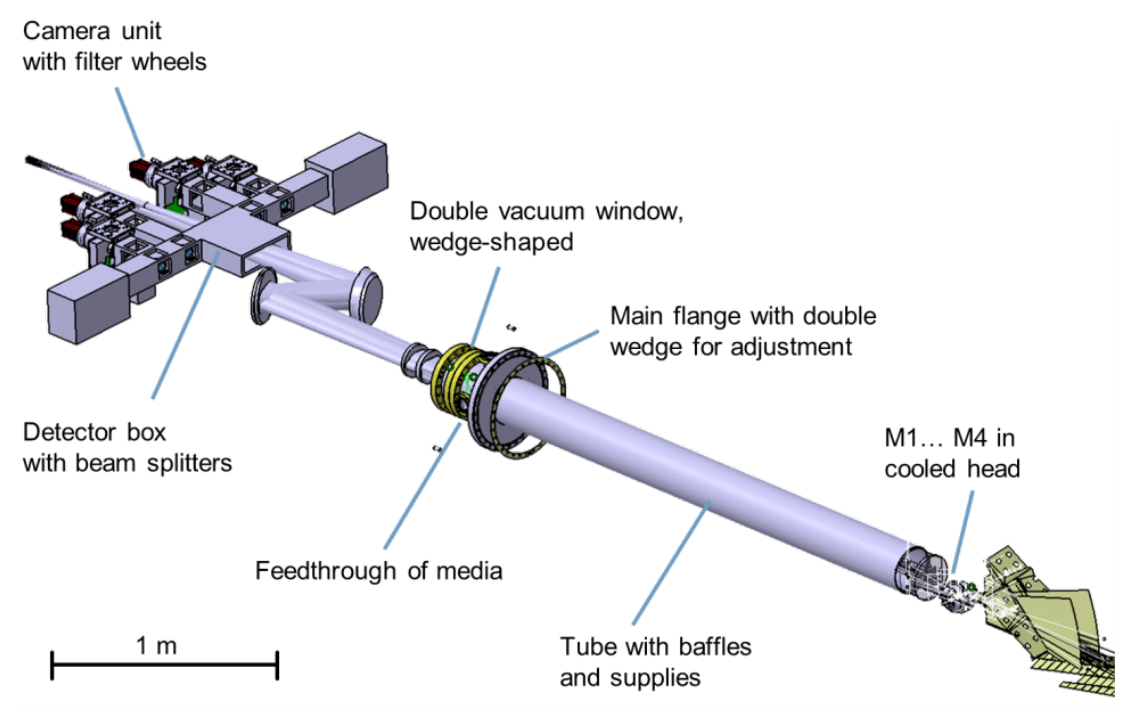

Fig. 3 Overview on the overall concept of the endoscopes.

The light to be observed is collected by a rotatable first mirror (M1) located behind an aperture. Via three subsequent mirrors (M2-M4) it is transmitted through a vacuum window at the end of a tube to a second set of mirrors (M5-M6). Within the detector box the light is divided into ultraviolet (350 nm - $550 \mathrm{~nm})$, visible (550 nm - $950 \mathrm{~nm})$ and infrared (950 nm - $7000 \mathrm{~nm}) \mathrm{com}$ ponents. Cameras equipped with narrow band interference filters similar to the endoscope for JET [6] as well as spectrometers linked via fibre optics are foreseen. 


\subsection{Optic layout}

The optical concept of the tube front end is determined by the requirements of transferring the light towards the rear end and tilting the first mirror. Furthermore, in order to be able to avoid collisions in the tight space around the endoscopes at Wendelstein 7-X, detector boxes should allow for rotation around the tube axis. All this should have no effect on the image quality. The solution found (figure 4) is to correct the distortions caused by mirror 2 using mirror 4 in the front end while mirrors 5 and 6 determine the focal length.

For safety reasons a double window provides the vacuum barrier. In view of the range of wavelengths to be passed $\mathrm{CaF}_{2}$ was selected for the windows. By wedge-like shaping ghost images are deflected out of the field of the subsequent mirror.

At the position of the pupil in the detector box (figure 4) a pyramidal mirror splits light into two identical fractions. With respect to transmission/reflection properties available for dichroic beam splitters, in both branches the ultraviolet (UV) fraction followed by the visible fraction (VIS) are deflected leaving the near to medium infrared (IR) only in the straight path. The total number of outputs is chosen such as to observe two spectral lines and two spectra (overview and high resolution) in UV; three spectral lines (atomic helium for profile measurements) and a spectrum in VIS; and one line and one spectrum in IR range. One more spectrum can be recorded via fibres located in the edge of the pyramidal mirror and linked to an overview spectrometer. This can be used to verify the optical emission in the bandwidth of the narrowband filters. For start-up the observation of hydrogen, helium, carbon, neon, nitrogen, and oxygen is foreseen. Another fibre in the pyramidal mirror allows for input of light which propagates the full path towards the first mirror and back to the detectors for relative intensity calibration.

The transmission without intensity splitting amounts to approximately $38 \%$ taking into account 6 aluminium coated mirrors (90\% each), $2 \mathrm{CaF}_{2}$ windows (95\% each), and 1 dichroic filter (80\%).

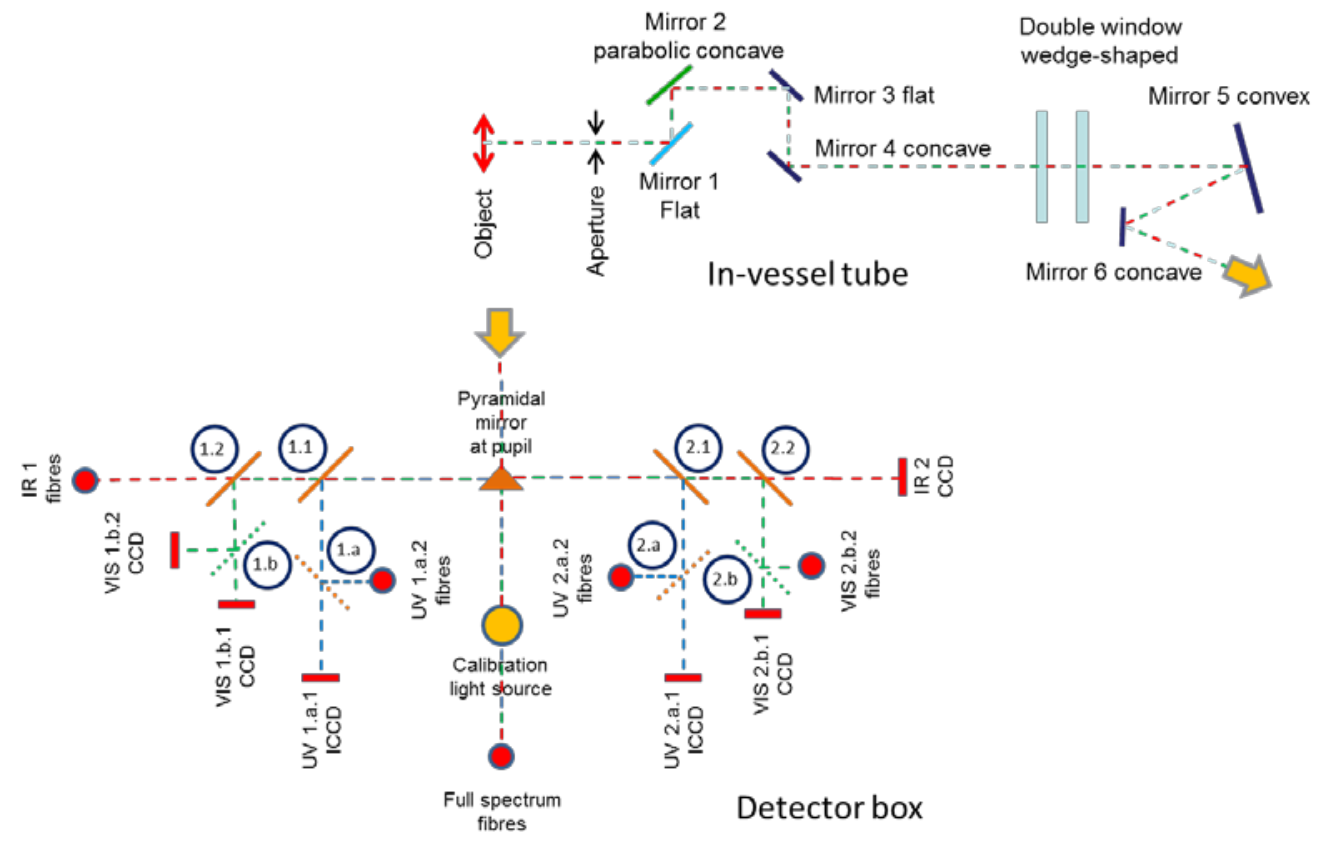

Fig. 4 Optic layout of the endoscopes subdivided into in-vessel tube (top right) and detector box (bottom left). 


\subsection{Endoscope components}

The endoscope head contains four mirrors allowing the first one to rotate. Heat radiation of up to $100 \mathrm{~kW} / \mathrm{m}^{2}$ from the plasma requires cooling of the head. The interior is protected by a casing with an aperture of $4 \mathrm{~mm}$ by $4 \mathrm{~mm}$. The first mirror together with casing and aperture is mounted on a piezoelectric drive. This unit combines functionalities of choosing the field of view, shutter, and back reflection of calibration light by corresponding rotation. All mirrors are equipped with adjustable three-point supports.

The endoscope tube has several baffles and broadband anti-reflective coating inside in order to reduce stray light propagation. The tube flange has to transfer full weight and momentum of the endoscope to the cryostat flange. Two rotatable wedge-shaped rings provide an angular adjustment compensating tolerances in the cryostat geometry.

The camera units have adjustable mounts, and similar to the JET endoscopes [6] consist of two filter wheels with 6 narrow band interference filters or 6 attenuation filters, respectively, a taper and - for the UV range - an image intensifier. Filter wheels are controlled by a motor via worm gear. The high voltage supply for the image intensifier is attached to the camera unit. Control of filter selection and acceleration voltage is integrated into the camera control unit.

\section{Summary}

Detailed local observation of the island divertor plasma will provide important information for understanding corresponding plasma-wall interactions which is crucial for successful operation of the advanced stellarator Wendelstein 7-X. A versatile optimized mirror based endoscope system allows simultaneous investigation of temperature and density profiles, impurities, hydrogen recycling, and transient heat load deposition.

\section{Acknowledgments}

This work has partly been carried out within the framework of the EUROfusion Consortium and has received funding from the Euratom research and training programme 2014-2018 under grant agreement No 633053. The views and opinions expressed herein do not necessarily reflect those of the European Commission.

\section{References}

[1] T. Klinger et al., Towards assembly completion and preparation of experimental campaigns of Wendelstein 7-X in the perspective of a path to a stellarator fusion power plant, Fus. Eng. Design 88, 461 (2013)

[2] S. Bosch, et al., Transition from Construction to Operation Phase of the Wendelstein 7-X Stellarator, IEEE Transactions on Plasma Science 42, 432 (2014)

[3] O. Neubauer et al., Diagnostic setup for investigation of plasma wall interactions at Wendelstein 7$X$, submitted to Fus. Eng. Design (2015)

[4] König, R., The divertor program in stellarators, Plasma Phys. Control. Fusion 44 (2002) 23652422

[5] Grigull, P., Divertor operation in stellarators: results from W7-AS and implications for future devices, Fusion Engineering and Design 66-68 (2003) 49-58

[6] Huber, A., Development of a mirror-based endoscope for divertor spectroscopy on JET with the new ITER-like wall, Review of Scientific Instruments, 83, 10D511 (2012) 